\title{
Association between small heat shock protein B11 and the prognostic value of $M G M T$ promoter methylation in patients with high-grade glioma
}

\author{
Wen Cheng, MM, ${ }^{1,6}$ Mingyang Li, MM, ${ }^{2,3,6}$ Yang Jiang, MM, ${ }^{1,6}$ Chuanbao Zhang, MD, PhD, ${ }^{2,6}$ \\ Jinquan Cai, MD, PhD, ${ }^{4,6}$ Kuanyu Wang, MM, ${ }^{5,6}$ and Anhua Wu, MD, PhD ${ }^{1,6}$
}

Department of Neurosurgery, ${ }^{1}$ The First Hospital of China Medical University, Shenyang; ${ }^{4}$ The Second Affiliated Hospital of Harbin Medical University, Harbin; ${ }^{5}$ The First Affiliated Hospital of Dalian Medical University, Dalian; ${ }^{3}$ Beijing Tiantan Hospital, Capital Medical University; ${ }^{2}$ Beijing Neurosurgical Institute; and ${ }^{6}$ Chinese Glioma Cooperative Group, Beijing, China

\begin{abstract}
OBJECTIVE This study investigated the role and prognostic value of heat shock proteins (HSPs) in glioma.
METHODS Data from 3 large databases of glioma samples (Chinese Glioma Genome Atlas, Repository for Molecular Brain Neoplasia Data, and GSE16011), which contained whole-genome messenger RNA microarray expression data and patients' clinical data, were analyzed. Immunohistochemical analysis was performed to validate protein expression in another set of 50 glioma specimens.
\end{abstract}

RESULTS Of 28 HSPs, 11 were overexpressed in high-grade glioma (HGG) compared with low-grade glioma. A univariate Cox analysis revealed that HSPB11 has significant prognostic value for each glioma grade, which was validated by a Kaplan-Meier survival analysis. HSPB11 expression was associated with poor prognosis and was independently correlated with overall survival (OS) in HGG. This study further explored the combined role of HSPB11 and other molecular markers in $\mathrm{HGG}$, such as isocitrate dehydrogenase 1 (IDH1) mutation and $\mathrm{O}^{6}$-methylguanine-DNA methyltransferase (MGMT) promoter methylation status. HSPB11 expression was able to refine the prognostic value of IDH1 mutation in patients with HGG. However, when combined with MGMT promoter methylation status, among patients with a methylated MGMT promoter, those with lower levels of HSPB11 expression had longer OS and progression-free survival than patients with higher levels of HSPB11 expression or with an unmethylated MGMT promoter. Moreover, within the MGMT promoter methylation group, patients with low levels of HSPB11 expression were more sensitive to combined radiochemotherapy than those with high levels of HSPB11 expression, which may explain why some patients with HGG with a methylated MGMT promoter show tolerance to radiochemotherapy.

CONCLUSIONS HSPB11 was identified as a novel prognostic marker in patients with HGG. Together with MGMT promoter methylation status, HSPB11 expression can predict outcome for patients with HGG and identify those who would most benefit from combined radiochemotherapy.

http://thejns.org/doi/abs/10.3171/2015.5.JNS142437

KEY WORDS HSPB11; prognosis; MGMT promoter methylation; glioma; oncology

$\mathrm{G}$ LIOMA is the most common type of tumor in the CNS. The WHO classifies glioma on a scale of Grades I-IV, according to histopathologic features. ${ }^{28}$ Grades III and IV are progressive, resistant to treatment, and grouped together as high-grade glioma (HGG) for the purposes of clinical management. Glioblastoma and anaplastic glioma comprise the majority of HGG cases. Since the incorporation of alkylating agents such as temozolomide (TMZ) into routine chemotherapy, the prognosis for patients with HGG has significantly improved. The epigenetic silencing of $\mathrm{O}^{6}$-methylguanineDNA methyltransferase (MGMT) (which encodes a DNA repair protein) by promoter methylation is associated with favorable prognosis in patients with HGG.$^{35,40,41}$ Numerous studies have also shown that MGMT promoter methylation can be used to predict the extent to which patients

ABBREVIATIONS CGGA = Chinese Glioma Genome Atlas; GBM = glioblastoma; $\mathrm{GO}$ = gene ontology; GSEA = Gene Set Enrichment Analysis; HGG = high-grade glioma; $\mathrm{HSP}=$ heat shock protein; IDH1 = isocitrate dehydrogenase 1; KPS = Karnofsky Performance Scale; LGG = low-grade glioma; $M G M T=0^{6}$-methylguanine-DNA methyltransferase; $m R N A$ = messenger RNA; OS = overall survival; PCR = polymerase chain reaction; PFS = progression-free survival; REMBRANDT = Repository for Molecular Brain Neoplasia Data; SAM = significance analysis of microarrays; TMZ = temozolomide.

SUBMITTED October 25, 2014. ACCEPTED May 7, 2015.

INCLUDE WHEN CITING Published online November 6, 2015; DOI: 10.3171/2015.5.JNS142437. 
will benefit from chemotherapeutic alkylating agents. ${ }^{18}$ However, even among patients with a methylated MGMT promoter, resistance to chemotherapy and unfavorable prognosis after standard treatment are observed. This suggests that additional factors predict response to treatment and determine clinical outcome.

Current cancer treatments aim to undermine cell viability and thereby enhance the cellular stress response. Heat shock proteins (HSPs) are highly conserved proteins present in normal cells. HSP expression is upregulated in response to endogenous and exogenous stress. ${ }^{23}$ HSPs have various functions, including protection against cytotoxic stress and maintenance of cellular homeostasis. High levels of HSP expression are required for cells to survive lethal conditions such as radiation, exposure to chemical agents, and hypoxia. ${ }^{10,16,23,25,26}$ Upregulation of HSP expression has been detected in many types of can$\operatorname{cer}^{13}$ and is often associated with poor clinical outcome and resistance to chemotherapy. ${ }^{6,47}$ Previous studies have also reported high levels of HSPs (including members of the small HSP family, a group of chaperones with monomeric molecular weights ranging from 12 to $43 \mathrm{kD}$ and heat shock-like properties) in malignant glioma. ${ }^{1,17,20}$ However, there is limited information on the role and predictive value of HSPs in glioma.

In this study, messenger RNA (mRNA) microarray expression data from 3 independent, large databases of samples comprising all grades of glioma were analyzed. The expression level and prognostic value of HSPs in glioma were evaluated. HSPBII was the only HSP that had significant prognostic value for Grades II-IV. Its expression was associated with poor prognosis and served as an independent prognostic factor for patients with HGG. HSPB11 also enhanced the predictive value of $M G M T$ promoter methylation status in patients with HGG.

\section{Methods}

\section{Patients and Samples}

A total of 295 samples from the Chinese Glioma Genome Atlas (CGGA) were included in this study as the discovery set, which contained whole-genome mRNA expression microarray data and corresponding clinical information (Supplemental Table S1). Tumor samples were obtained from patients with newly diagnosed glioma who were treated by the CGGA group. Each sample underwent histological analysis, results of which were independently confirmed by 2 neuropathologists based on the 2007 WHO classification guidelines. ${ }^{28}$ All patients provided written informed consent. The study was approved by the ethics committees of participating hospitals. The Repository for Molecular Brain Neoplasia Data (REMBRANDT; National Cancer Institute, http://caintegrator.nci.nih.gov/ rembrandt) and GSE16011 (Gene Expression Omnibus; National Center for Biotechnology Information, http:// www.ncbi.nlm.nih.gov/geo/query/acc.cgi?acc=GSE16011) databases were used as validation data sets.

\section{Immunohistochemical Analysis}

HSPB11 immunostains were done using formalinfixed, paraffin-embedded tissues. Four-micrometer-thick sections were cut from each paraffin block, dewaxed in xylene, rinsed in graded ethanol, and rehydrated in double-distilled water. The sections were then treated with $3 \% \mathrm{H}_{2} \mathrm{O}_{2}$ for 5 minutes at room temperature to block endogenous peroxidase activity. For antigen retrieval, slides were pretreated by steaming in sodium citrate buffer $(10$ $\mathrm{mM}$ sodium citrate, $\mathrm{pH}$ 6.0) for 15 minutes at $100^{\circ} \mathrm{C}$. After washing with phosphate-buffered saline for 3 minutes, the sections were manually immunostained with an antihuman HSPB11 rabbit polyclonal antibody (Proteintech, 15732-1-AP) at 1:50 dilution with standard avidin-biotinperoxidase. Staining for HSPB11 was scored manually for the percentage of positive cells. We chose the 5 most heavily stained high-resolution fields under $\times 200$ magnification, determined the percentage of positive cells, and calculated the average of the percentages. Five categories were used to assess the staining intensity in cells: 0 , no positive staining; $1,1 \%-10 \%$ positive staining; $2,11 \%$ $30 \%$ positive staining; $3,31 \%-50 \%$ positive staining; and $4,>50 \%$ positive staining.

\section{Evaluation of Isocitrate Dehydrogenase 1 Mutation Status by DNA Pyrosequencing}

Genomic DNA was isolated from frozen tumor samples using the QIAamp DNA Mini Kit (QIAGEN). The genomic region spanning wild-type R132 of isocitrate dehydrogenase $1(\mathrm{IDH})$ was analyzed by pyrophosphate sequencing using the following primers: 5'-GCT TGT GAG TGG ATG GGT AAA AC-3' and 5'-biotin-TTG CCA ACA TGA CTT ACT TGA TC-3'. The polymerase chain reaction (PCR) analysis was performed in duplicate in a 40- $\mu \mathrm{l}$ reaction volume, containing $1 \mu \mathrm{l}$ of $10 \mu \mathrm{M}$ each forward and reverse primer, $4 \mu \mathrm{l} 10 \times$ buffer for $3.2 \mu \mathrm{l}$ of $2.5 \mathrm{mM}$ deoxynucleoside triphosphate, $2.5 \mathrm{U}$ hotstart Taq (Takara), and $2 \mu \mathrm{l}$ of $10 \mu \mathrm{M}$ DNA. The PCR conditions were as follows: $95^{\circ} \mathrm{C}$ for 3 minutes, 50 cycles of $95^{\circ} \mathrm{C}$ for 15 seconds, $56^{\circ} \mathrm{C}$ for 20 seconds, $72^{\circ} \mathrm{C}$ for 30 seconds, and $72^{\circ} \mathrm{C}$ for 5 minutes (ABI PCR system 9700). Singlestranded DNA was purified from the total PCR products and subjected to pyrosequencing on a PyroMark Q96 ID System (QIAGEN) using the primer 5'-TGG ATG GGT AAA ACC T-3' and EpiTect Bisulfite Kit (QIAGEN).

\section{Evaluation of MGMT Promoter Methylation by DNA Pyrosequencing}

The MGMT promoter methylation status was detected by DNA pyrosequencing as previously described. ${ }^{43,44}$ Bisulfite modification of DNA was performed using the EpiTect Bisulfite Kit. Sequences of the primer set used to amplify the $M G M T$ promoter region were as follows: 5'-GTT TYG GAT ATG TTG GGA TA-3' and 5'-biotinACC CAA ACA CTC ACC AAA TC-3'. Pyrosequencing analysis was performed by Gene Tech. Obtained methylation values were averaged across $7 \mathrm{CpG}$ loci within the $M G M T$ promoter. Methylation was defined as samples with an average methylation value $>10 \%$.

\section{Gene Ontology Analysis of Differential Genes in HGG With Methylated MGMT Promoter}

Patients with HGG who had a methylated MGMT pro- 
moter were stratified into 2 groups based on their median HSPB11 expression level. A significance analysis of microarrays (SAM) was performed to identify differential probes with threshold of false discovery rate $=0.1$ and number of permutations $=100$. A gene ontology $(\mathrm{GO})$ analysis of different probes was performed using the Database for Annotation, Visualization and Integrated Discovery (Laboratory of Immunopathogenesis and Bioinformatics, SAIC-Frederick, Inc., http://david.abcc.ncifcrf. gov/home.jsp) functional annotation tool..$^{22}$

\section{Gene Set Enrichment Analysis}

To obtain functional information on HSPB11, Gene Set Enrichment Analysis (GSEA) (Broad Institute, http:// www.broadinstitute.org/gsea/index.jsp) was performed as previously described, ${ }^{36}$ which identified gene sets showing statistically significant, concordant differences between the 2 biological states based on the C5.bp GO gene set collection.

\section{Statistical Analysis}

SPSS (SPSS, Inc.) and GraphPad Prism 6 (GraphPad Software, Inc.) software were used for statistical analyses. Differences in HSP expression levels between groups were compared using Student's t-test in microarray data and the chi-square test in immunohistochemical analysis. The normalization of gene expression was performed using the "normalize genes" and "normalize arrays" options in Cluster 3.0 (Human Genome Center, Institute of Medical Science, University of Tokyo) before transforming expression information into a heat map. Overall survival (OS) and progression-free survival (PFS) were used as clinical end points. The former was calculated from the date of diagnosis until death or the end of follow-up; the latter was defined as the time between diagnosis and the first unequivocal clinical or radiological sign of disease progression. A Cox regression analysis was performed to evaluate the prognostic value of HSPs in glioma. A Kaplan-Meier survival analysis was used to estimate the survival distribution, and the log-rank test was used to evaluate differences between stratified groups, using the median value as the cutoff. Statistical significance was defined as a 2-tailed $\mathrm{p}$ value $<0.05$.

\section{Results}

\section{Expression of HSPs Is Dysregulated in Glioma}

Of 295 glioma samples in the CGGA that were included in this study, $119(40.34 \%)$ samples were low-grade glioma (LGG) and 176 (59.66\%) were HGG. The expression of 28 HSPs was compared between LGG and HGG cases using Student's t-test. A total of 18 HSPs were differentially expressed between LGG and HGG: HSPB1, HSPE1, HSPA6, HSPB6, HSPB11, HSPA4, HSPA5, HSP9OB1, HSPB7, HSPA9, and HSP9OAAl were upregulated in HGG as compared with LGG (p < 0.05), whereas $H S P B 8$, HSPA12A, HSPA2, HSP90AB1, HSPB5, HSPA12B, and $H S P B 9$ were downregulated in LGG $(\mathrm{p}<0.05)$. Prior to transforming HSP expression levels into a heat map for visualization (Fig. 1A), the "normalize genes" and "normalize arrays" options in Cluster 3.0 were performed for the purposes of normalization and increasing the comparability among these genes. A summary of mean differences and $p$ values for each HSP based on the t-test in the CGGA database is shown in Supplemental Table S2. These findings suggest that specific HSPs play important roles in glioma progression and pathogenesis.

\section{High HSPB11 Expression Level Is Associated With Unfavorable Prognosis in Glioma}

To evaluate the prognostic value of HSPs in patients with glioma, a univariate Cox regression analysis was performed for each grade; the results are summarized in Table 1. Of the 28 HSPs, only HSPB11 was related to prognosis for each glioma grade (Grade II: $\mathrm{HR}=2.5958, \mathrm{p}$ $=0.0459$; Grade III: HR $=2.4233, \mathrm{p}=0.0025$; and Grade IV: $\mathrm{HR}=1.5817, \mathrm{p}=0.0104)$.

The prognostic value of $H S P B 11$ was further evaluated based on the median expression level using the KaplanMeier survival analysis and log-rank test. Patients with Grade II-IV HGG had different outcomes depending on HSPB11 expression level: patients with low HSPB11 expression level had longer OS and PFS than those in whom HSPB11 expression was upregulated (Fig. 1B-G). Similar analyses in the 2 validation sets (REMBRANDT and GSE16011) confirmed the results that HSPB11 overexpression confers a poor prognosis (Supplemental Fig. S1). Both the univariate Cox and Kaplan-Meier analyses showed that HSPB11 expression level was significantly associated with prognosis in patients with glioma.

\section{Expression Level of HSPB11 Is Associated With Progressive Glioma Malignancy}

According to the expression data in the CGGA database, HSPB11 expression was notably decreased in Grade II relative to Grades III ( $p<0.0001)$ and IV $(\mathrm{p}<0.0001)$ glioma (Fig. 2A), although expression levels were similar between Grades III and IV ( $\mathrm{p}=0.4669)$ (Fig. 2A), which are both considered HGG. A comparison of HSPBI1 expression levels in LGG and HGG based on the CGGA and validated with the REMBRANDT and GSE16011 data sets revealed that HSPBII was upregulated in HGG (Fig. 2B-D).

We further explored the expression level of HSPB11 in glioma specimens from 50 patients (20 with Grade II, 15 with Grade III, and 15 with glioblastoma (GBM) using immunohistochemical analysis (Fig. 2E-G). In concordance with the findings above, HSPBII showed a higher expression status in HGG than in LGG ( $p<0.0001$, chi-square test), whereas no significant difference was observed between Grades III and IV ( $p=0.4151$, chi-square test) (Supplemental Table S3). The concordance in the results from the 3 independent data sets and the immunohistochemical analysis confirmed that a strong correlation exists between HSPB11 expression level and glioma malignancy.

\section{Level of HSPB11 Expression Is an Independent Prognostic Factor in HGG}

Given that HSPBII is highly expressed in HGG, the prognostic value of HSPB11 expression level in patients with HGG was investigated. Dichotomization was used to 

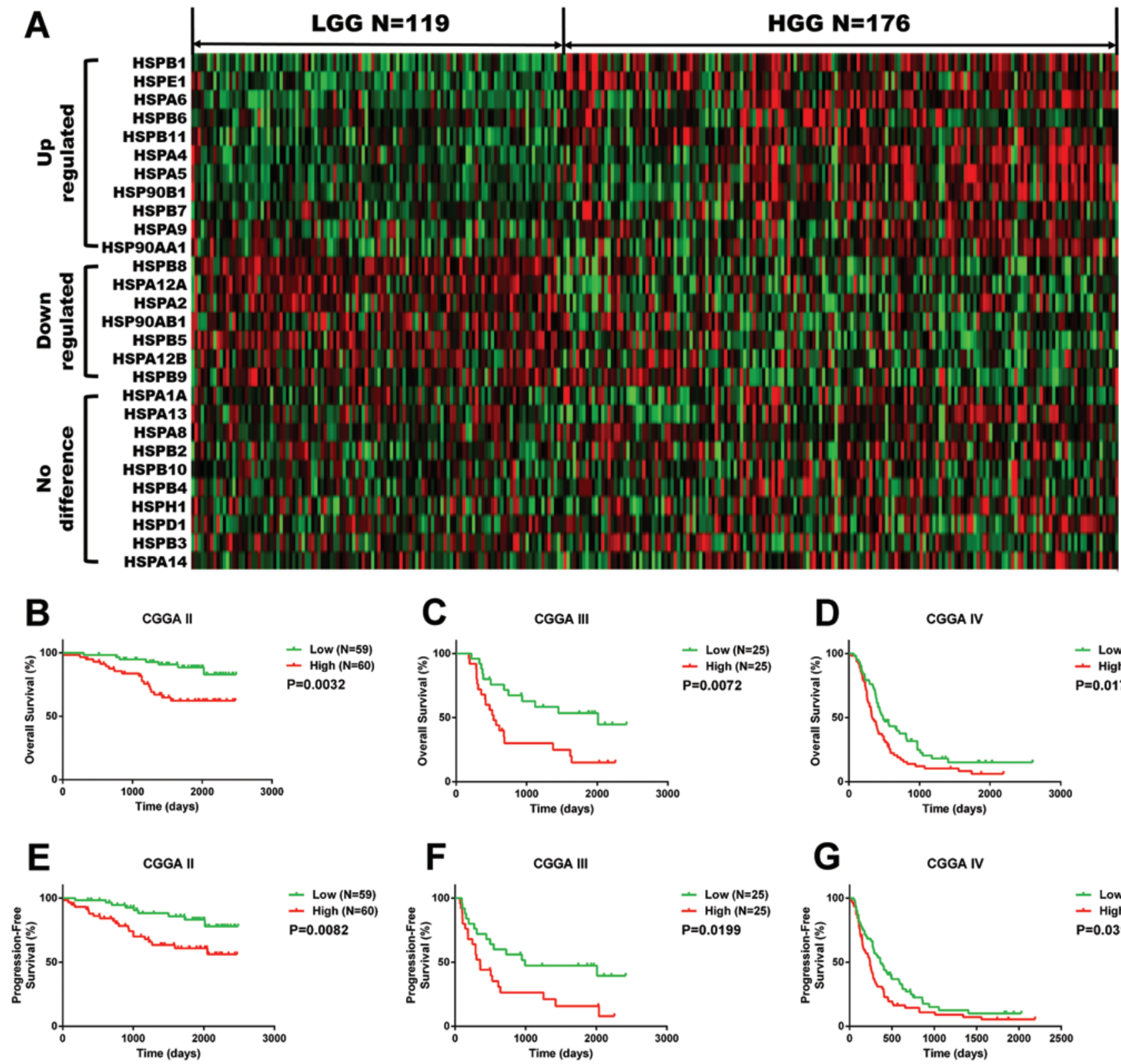

FIG. 1. A heat map is shown, depicting HSP expression levels in LGG versus HGG. Genes were clustered into 3 groups (upregulated in HGG, downregulated in HGG, and no significant difference). Differences in expression levels were evaluated with the Student t-test (A). Survival analysis was performed to assess the prognostic value of HSPB11 for each glioma grade in the CGGA database. The discriminative power of HSPB11 was tested using the Kaplan-Meier analysis and log-rank test. HSPB11 downregulation was associated with prolonged OS (B-D) and PFS (E-G) for each grade. Figure is available in color online only.

classify samples into 2 groups based on median HSPB11 expression level in patients with HGG. In the CGGA and the 2 validation data sets, the prognostic value of HSPB11 was enhanced in patients with HGG, and patients with high levels of HSPB11 expression had significantly reduced survival time compared with those with low expression levels (Figs. 3A and B and Supplemental Fig. S2). Next, a univariate Cox regression analysis was performed using clinical variables for 176 patients with $\mathrm{HGG}$ in the CGGA. Age, tumor grade, Karnofsky Performance Scale (KPS) score, radiotherapy, chemotherapy, and HSPB11 expression level were all associated with OS. Then, multivariate Cox regression analysis, evaluating factors that contribute to OS, found that HSPB11 expression level was independently correlated with OS in patients with HGG $(\mathrm{HR}=2.155, \mathrm{p}=0.008)$ (Table 2$)$ when age, tumor grade, KPS score, radiotherapy, and chemotherapy were considered.

\section{The Expression Level of HSPB11 Confers Different Responses to Radiochemotherapy}

Because there are great disparities among patients' re- 
TABLE 1. Correlation of HSPs with patients' prognoses in each grade of CGGA database

\begin{tabular}{|c|c|c|c|c|c|c|}
\hline \multirow[b]{2}{*}{ HSP } & \multicolumn{2}{|c|}{ WHO Grade II } & \multicolumn{2}{|c|}{ WHO Grade III } & \multicolumn{2}{|c|}{ WHO Grade IV } \\
\hline & HR & $p$ Value & HR & $p$ Value & HR & $p$ Value \\
\hline HSP90AA1 & 0.9783 & 0.9607 & 0.9020 & 0.7146 & 1.1704 & 0.4612 \\
\hline HSP90AB1 & 0.4575 & 0.1022 & 0.3993 & 0.0106 & 0.9123 & 0.6250 \\
\hline HSP90B1 & 1.3407 & 0.5623 & 0.8398 & 0.6413 & 1.0635 & 0.6957 \\
\hline HSPA12A & 0.6434 & 0.1781 & 0.8364 & 0.3631 & 0.8454 & 0.2159 \\
\hline HSPA12B & 2.0246 & 0.0364 & 0.8616 & 0.4899 & 0.8132 & 0.1248 \\
\hline HSPA13 & 0.4625 & 0.0894 & 0.7768 & 0.3238 & 1.0879 & 0.6379 \\
\hline HSPA14 & 0.6544 & 0.4419 & 0.7661 & 0.4179 & 1.1596 & 0.3762 \\
\hline HSPA1A & 1.0449 & 0.8717 & 0.9845 & 0.9333 & 1.0717 & 0.5643 \\
\hline HSPA2 & 1.1505 & 0.4324 & 0.9972 & 0.9791 & 0.9954 & 0.9450 \\
\hline HSPA4 & 3.1513 & 0.1579 & 2.5368 & 0.1687 & 0.7745 & 0.2737 \\
\hline HSPA5 & 0.5610 & 0.3477 & 2.0172 & 0.0754 & 1.0664 & 0.6362 \\
\hline HSPA6 & 1.0381 & 0.8067 & 1.2289 & 0.0366 & 1.0956 & 0.1357 \\
\hline HSPA8 & 0.2978 & 0.0583 & 0.2234 & 0.0075 & 0.8206 & 0.2052 \\
\hline HSPA9 & 0.9256 & 0.8319 & 0.4834 & 0.0517 & 0.8425 & 0.3083 \\
\hline HSPB1 & 1.0139 & 0.9506 & 1.2685 & 0.1364 & 1.1284 & 0.2157 \\
\hline HSPB2 & 0.8318 & 0.3902 & 0.9614 & 0.8006 & 0.9815 & 0.8321 \\
\hline HSPB3 & 0.8558 & 0.1884 & 0.9369 & 0.4777 & 1.0202 & 0.7304 \\
\hline HSPB4 & 1.3117 & 0.4454 & 1.3233 & 0.3473 & 0.9395 & 0.5592 \\
\hline HSPB5 & 1.1547 & 0.5142 & 0.7640 & 0.0603 & 0.8664 & 0.0539 \\
\hline HSPB6 & 1.0599 & 0.8742 & 1.5056 & 0.0150 & 1.2719 & 0.0265 \\
\hline HSPB7 & 0.9467 & 0.8424 & 1.5297 & 0.0013 & 0.9861 & 0.8796 \\
\hline HSPB8 & 0.9508 & 0.7982 & 0.8590 & 0.1675 & 0.9358 & 0.3309 \\
\hline HSPB9 & 1.2264 & 0.6237 & 0.5693 & 0.2255 & 0.8419 & 0.3226 \\
\hline HSPB10 & 0.9718 & 0.9346 & 0.7222 & 0.1807 & 1.0363 & 0.6950 \\
\hline HSPB11 & 2.5958 & 0.0459 & 2.4233 & 0.0025 & 1.5817 & 0.0104 \\
\hline HSPD1 & 1.7655 & 0.3018 & 0.3246 & 0.0337 & 1.1139 & 0.5477 \\
\hline HSPE1 & 5.2043 & 0.0062 & 0.8487 & 0.6194 & 0.9666 & 0.8619 \\
\hline HSPH1 & 0.8539 & 0.5270 & 0.7479 & 0.1721 & 1.2608 & 0.0932 \\
\hline
\end{tabular}

sponses to treatment strategy, we attempted to explore the relationship between HSPB11 expression level and patients' responses to chemotherapy, which is widely used in routine clinical management. Patients with HGG were classified into 2 groups according to the median expression level of HSPB11. Survival analysis revealed that patients with lower levels of HSPB11 expression who received radiochemotherapy survived significantly longer than those who received radiotherapy alone $(\mathrm{p}=0.0013)$ (Supplemental Fig. S3A). However, in patients for whom HSPB11 was upregulated, the difference in prognosis between radiotherapy and radiochemotherapy groups was not statistically significant $(p=0.1278)$ (Supplemental Fig. S3B).

\section{The Prognostic Association Between HSPB11 Expression Level and IDH1 Mutation Status in HGG}

The IDHI mutation status and MGMT promoter methylation status have been identified as stable prognostic and predictive indicators for patients with HGG in various studies. ${ }^{18,35,40,41,45}$ The IDHI mutation status and MGMT promoter methylation status were analyzed in 147 randomly selected HGG samples by DNA pyrosequencing as described above. A total of 44 samples contained an IDH1 mutation, whereas 103 samples contained wild-type IDH1. The survival time of patients with an IDHI mutation was significantly longer than that of patients without an IDHI mutation (Supplemental Fig. S4), which was consistent with previous reports. Afterward, IDH1 mutation status combined with HSPB11 expression level was used for stratification, classifying patients into 4 subgroups: 22 (15.0\%) samples with IDHI mutation/low HSPBII expression, $22(15.0 \%)$ samples with $I D H 1$ mutation/high HSPB11 expression, 51 (34.7\%) samples with wild-type $I D H 1 /$ low HSPBII expression, and 52 (35.4\%) samples with wild-type $I D H 1 /$ high $H S P B 11$ expression. Survival analysis indicated that OS and PFS were significantly different among these 4 subgroups (Fig. 3C and D). Although there were no statistical differences in clinical outcome between 2 subgroups with equal IDHI mutation status, patients' survival times were notably different according to variable $H S P B I I$ expression between wild-type $I D H I$ subgroups. In addition, patients who had IDHI mutation/ low HSPB11 expression survived significantly longer than the 2 subgroups with wild-type IDHI (Fig. 3C and D). 

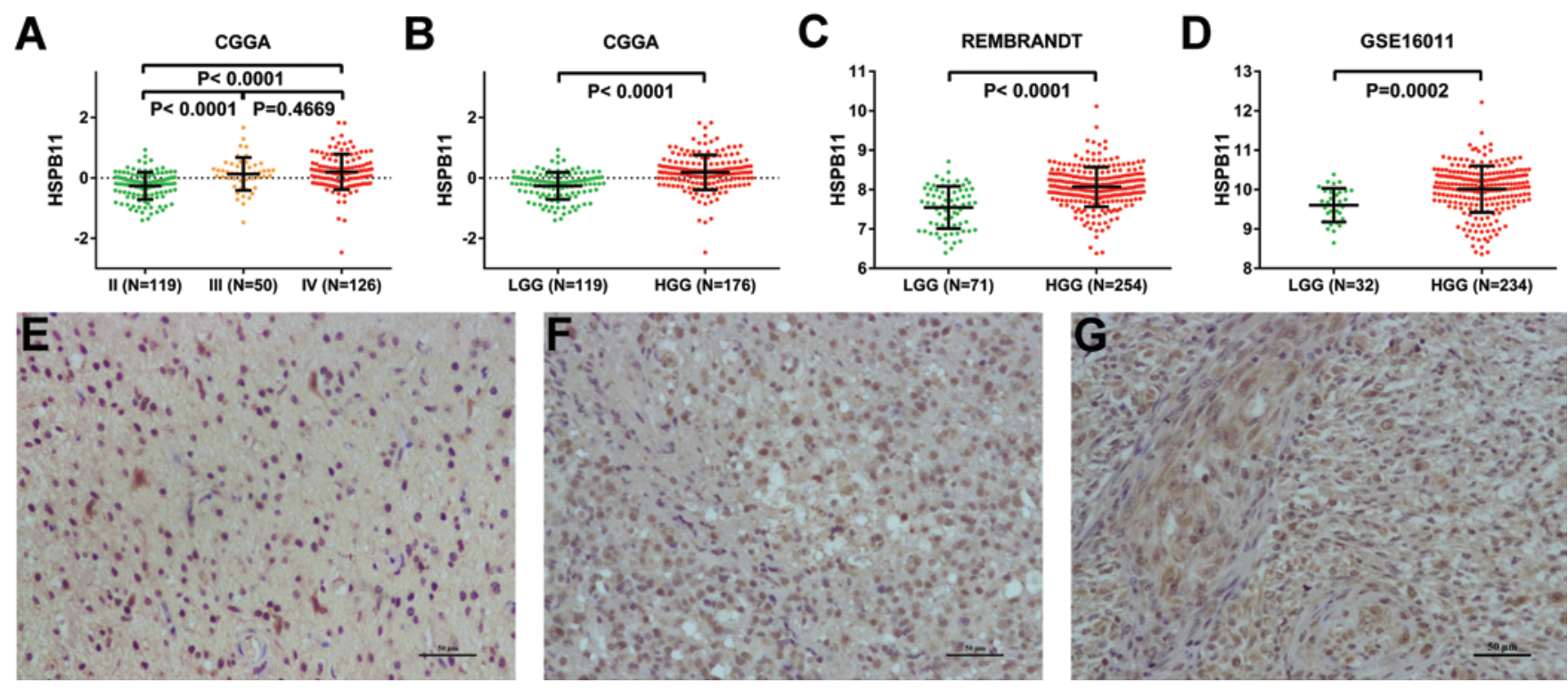

FIG. 2. Relationship between HSPB11 expression level and glioma grade analyzed using the Student t-test. HSPB11 expression was downregulated in Grade II compared with Grades III ( $<0.0001$ ) and IV ( $<<0.0001)$, whereas expression was similar between Grades III and IV $(p=0.4669)(A)$. Differences in HSPB11 expression between LGG and HGG were evaluated in the CGGA database and 2 independent validation data sets (B-D). Photomicrographs of immunohistochemical staining of HSPB11 in Grade II (E), III (F), and IV (G) are shown. Original magnification $\times 200$. Figure is available in color online only.

\section{Expression Level of HSPB11 Refines the Prognostic Value of MGMT Promoter Methylation in HGG}

Among 147 patients with HGG, DNA pyrosequencing data indicated that 76 patients had methylated $M G M T$ promoter, whereas 71 patients had unmethylated $M G M T$ promoter. Consistent with other studies, $M G M T$ promoter methylation had significant prognostic value in patients with HGG (Supplemental Fig. S5). Subsequently, MGMT promoter methylation status and HSPB11 expression level were incorporated into the classification, resulting in 4 subgroups: 38 (25.9\%) patients with methylated MGMT promoter/low HSPB11 expression, 38 (25.9\%) patients with methylated MGMT promoter/high HSPB11 expression, 35 (23.8\%) patients with unmethylated MGMT promoter/low HSPB11 expression, and $36(24.5 \%)$ patients with unmethylated MGMT promoter/high HSPB11 expression. Regardless of treatment, OS and PFS varied across subgroups $(\mathrm{p}=0.0006$ for OS and $\mathrm{p}=0.0012$ for PFS) (Fig. 3E and F). Patients with a methylated $M G M T$ promoter and low HSPBII expression had the best prognosis, with a median OS of 883 days and PFS of 546 days, which were longer than in the other 3 subgroups. In patients with a methylated $M G M T$ promoter, reduced $H S P B 11$ expression was associated with a better prognosis, whereas patients with high HSPB11 expression had similar prognoses to those with an unmethylated $M G M T$ promoter; in the latter, HSPB11 expression level did not affect prognosis.

The differences in prognosis among patients with a methylated MGMT promoter (who were presumed to be sensitive to chemotherapy) prompted an evaluation of the predictive value of $H S P B 11$ in these patients $(\mathrm{n}=76)$, who were stratified into 4 subgroups according to their HSPB11 expression level and treatment. The OS and PFS differed across subgroups $(p=0.0139$ for OS and $p=0.0163$ for PFS) (Fig. 3G and H). The longest OS and PFS rates were among patients with low HSPB11 expression who received combined radiochemotherapy (Fig. 3G and $\mathrm{H}$ ): their median OS and PFS were 970 and 716 days, respectively, compared with 455 and 341 days, respectively, among patients with low HSPB11 expression who received radiotherapy alone. Among patients with high HSPBII expression, there was no difference according to treatment received. The interesting findings regarding the relationship between HSPBII expression and MGMT promoter methylation status further highlighted the role of HSPB11 in predicting response to glioma treatment.

\section{Association of HSPB11 With Cell Cycle Regulation and the Maintenance of Genomic Integrity}

For patients with a methylated $M G M T$ promoter, responses to treatment varied according to HSPB11 expression levels; thus, the function of $H S P B 11$ was evaluated. A SAM was performed for high and low HSPBII expression subgroups of patients with HGG, with MGMT promoter methylation resulting in 294 probes (Supplemental Fig. S6). Differentially expressed genes were subjected to GO analysis, which revealed that HSPB11-expressing samples were associated with the cell cycle (Supplemental Table S4). GSEA was performed based on the level of HSPB11 expression (Supplemental Table S5). Genes associated with the maintenance of genomic integrity were enriched in the high HSPB11 expression group (Fig. 3I-M and Supplemental Table S4), suggesting that factors that affect cell cycle and maintain genomic integrity are responsible for patients' variable responses to treatment. 
A

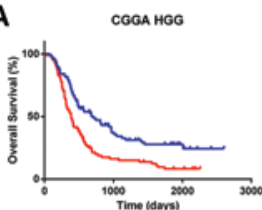

B
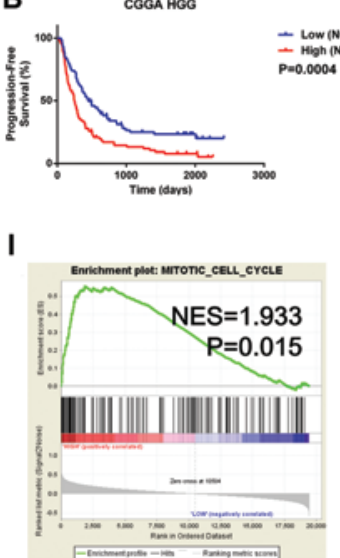

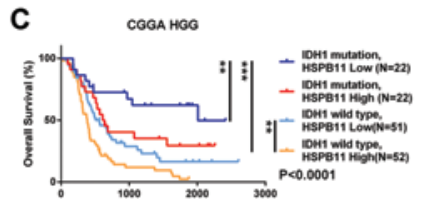

E

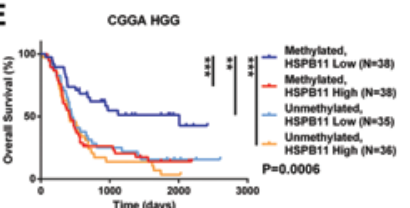

D COGA HOO
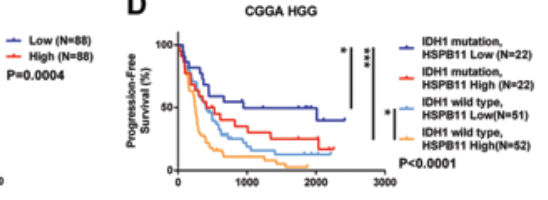

$\mathbf{F}$

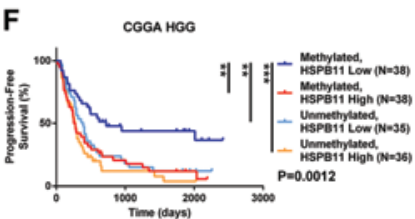

$\mathbf{K}$

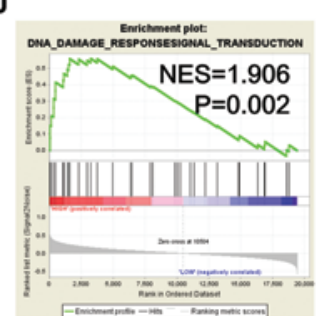

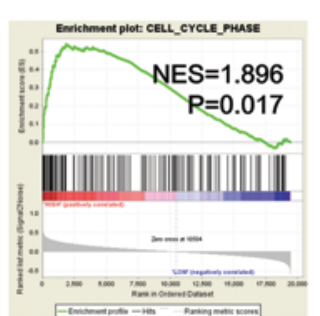

$\mathbf{L}$

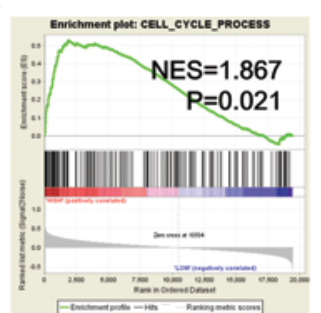

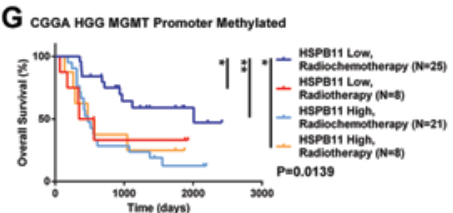

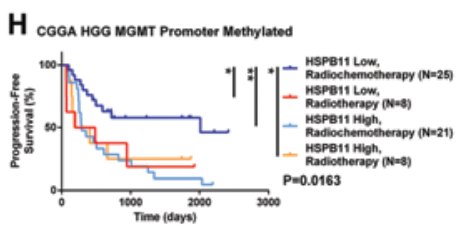

M

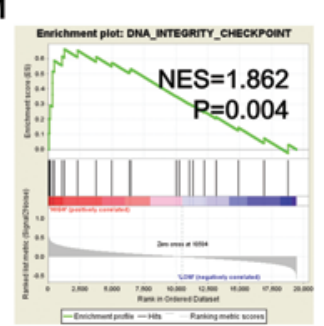

FIG. 3. Low levels of HSPB11 expression are associated with longer OS and PFS in patients with HGG (A and B). Although there was no statistical significance in survival time between 2 subgroups with equal IDH1 mutation status, patients with wild-type IDH1 had significantly different clinical outcomes according to their levels of HSPB11 expression (C and D). Patients with a methylated MGMT promoter and low levels of HSPB11 expression had the best prognoses; the prognoses of patients with a methylated MGMT promoter differed according to their HSPB11 expression levels. Patients with an unmethylated MGMT promoter showed no significant differences in prognosis (E and F). Survival analysis was performed for patients with HGG who had MGMT promoter methylation. Patients with low levels of HSPB11 expression who received radiochemotherapy had the best prognoses, whereas no differences were observed among the other groups ( $G$ and $H)$. An asterisk denotes statistical significance between 2 groups at the end point. GSEA was performed in patients with HGG who had MGMT promoter methylation (I-M). Genes associated with cell cycle and maintenance of genomic integrity were enriched in patients with high levels of HSPB11 expression. The horizontal bar in graded color from red to blue represents the rank ordering of patients based on decreasing levels of HSPB11 expression. Vertical black lines represent the projection of individual genes constituting relevant gene sets. Figure is available in color online only.

\section{Discussion}

Despite treatment advances in recent decades, the prognosis for patients with glioma remains poor, especially for HGG, with a median survival of 15 months and a 2-year survival rate of $27 \%$ after standard treatment consisting of maximal resection followed by adjuvant chemotherapy and radiotherapy. ${ }^{34}$ Due to its infiltrating nature, HGG cannot be completely excised and in most cases recurs locally. ${ }^{14}$ Given the high level of heterogeneity and inaccuracy of traditional clinicopathological factors such as age, grade, KPS score, and tumor resection for predicting prognosis, the identification of molecular markers in HGG that can help to distinguish among patients with different prognoses or clinical responses to specific therapies is essential.

HSPs are highly conserved proteins that are expressed at low levels under normal physiological conditions but are upregulated in response to cellular stress caused by heat, hypoxia, and toxicants. ${ }^{8,21,27,32}$ Cancer cells exist in a state of continuous stress induced by aberrant overproduction of oncoprotein, genomic instability, and local hypoxia and acidosis; HSPs are thus highly expressed in several cancer types, including malignant glioma..$^{23,33,46}$ In the present study, 11 HSPs were upregulated in HGG compared with LGG, suggesting that cells are under continuous stress in glioma, which may be linked to tumor malignancy. In this study, a univariate Cox analysis of 28 HSPs across glioma grades identified only $H S P B 11$ as having a significant prognostic value in each grade.

HSPB11 is a member of the small HSP protein family, and has intracellular localization, chaperoning, and oligomerization properties that are similar to HSP27.5 HSPB 11 is present only in tumor cells and its expression level in-

TABLE 2. Cox regression analyses of HSPB11 expression and other characteristics related to OS in HGG

\begin{tabular}{|c|c|c|c|c|}
\hline \multirow[b]{2}{*}{ Variable } & \multicolumn{2}{|c|}{$\begin{array}{l}\text { Univariate } \\
\text { Regression }\end{array}$} & \multicolumn{2}{|c|}{$\begin{array}{l}\text { Multivariate } \\
\text { Regression }\end{array}$} \\
\hline & $\mathrm{HR}$ & $p$ Value & $\mathrm{HR}$ & $p$ Value \\
\hline Sex & 0.962 & 0.826 & & \\
\hline Age & 1.021 & 0.005 & 1.006 & 0.558 \\
\hline Grade & 2.119 & $<0.001$ & 1.812 & 0.067 \\
\hline KPS score & 0.974 & $<0.001$ & 0.974 & 0.003 \\
\hline Resection & 0.779 & 0.203 & & \\
\hline Radiotherapy & 0.489 & 0.002 & 0.435 & 0.013 \\
\hline Chemotherapy & 0.463 & $<0.001$ & 0.602 & 0.079 \\
\hline HSPB11 expression & 1.826 & $<0.001$ & 2.155 & 0.008 \\
\hline
\end{tabular}


creases with the aggravation of cell anaplasia. ${ }^{4,30}$ Similar to what is observed for $H S P 27, H S P 72$, and $H S P 90$, a high $H S P B 11$ expression level was associated with poor prognosis for patients with glioma. ${ }^{19}$ However, only HSPB11 had significant prognostic value for each glioma grade in 3 large sample databases, which was verified using Cox regression and Kaplan-Meier survival analyses based on respective median expression levels. Because of the large differences exhibited in HSPBII expression levels among glioma grades, we focused on the survival analysis in specific groups of patients restricted by glioma grade. It was more reasonable to define an independent cutoff based on the median expression level of HSPBII in the corresponding analysis for dichotomization rather than set a uniform cutoff for all analyses. The cytoprotective effects of small HSPs rely on their molecular chaperone functions and interactions with components of the programmed cell death machinery. ${ }^{2,11}$ Under experimental conditions, similar to other small HSPs, HSPB11 overexpression can induce resistance to cell death by inhibiting mitochondrial cytochrome c release and caspase activation to suppress apoptosis. ${ }^{5,24,31}$ However, in cancer cells that have abnormally high HSP expression levels, the cytoprotective function is commandeered to protect cancer cells from the toxic effects of oncoprotein aggregation and chemo- and radiotherapy, which can explain why HSPs serve as negative prognostic markers. ${ }^{13,23}$

Based on the results of univariate Cox and KaplanMeier survival analyses, we indicated that the HSPB11 expression level was a determinant of patients' prognoses, with a high HSPB11 expression level associated with reduced survival in Grades II-IV and higher in HGG. It has been well established that multiple factors severely affect the prognosis for patients with glioma. Considering the combined effects of multiple factors, univariate Cox analysis and Kaplan-Meier survival analysis were not able to fully clarify the prognostic value of HSPBII in patients with HGG. Therefore, we conducted multivariate Cox regression analysis to verify the prognostic role of HSPB11, taking into consideration several critical clinical features, such as age, tumor grade, KPS score, radiotherapy, and chemotherapy. These results indicated that HSPBII is an independent prognostic factor in patients with HGG.

As one of the most popular molecular markers in glioma, IDHI mutation typically occurred in LGG, anaplastic glioma, and secondary GBMs, but rarely in primary GBM. ${ }^{3,42}$ Previous reports also identified IDHI mutation as a powerful prognostic marker in patients with $\mathrm{HGG}^{43,45}$ and found that $I D H 1$ mutation status was sufficient to delineate clinically distinct subclasses of glioma. ${ }^{37}$ However, patients exhibited various clinical outcomes, even with similar IDHI status; thus, studies were needed to further refine the prognostic value of IDHI. In our study, patients with wild-type $I D H I$ had significantly different clinical outcomes according to their HSPBII expression levels. Meanwhile, patients with $I D H 1$ mutation/low $H S P B 11$ expression always survived longer than patients with wild-type $I D H 1$. Our results indicated that, when incorporated with IDHI mutation status, HSPBII could be introduced for accurate prediction of prognosis, although further study is needed to clarify the hidden mechanism.
In routine clinical management, patients treated with alkylating agents such as TMZ have better prognoses than those receiving radiotherapy alone..$^{15}$ These agents cause cell death by cross-linking adjacent DNA strands. ${ }^{9} M G M T$ can rapidly reverse DNA alkylation; thus, the epigenetic silencing of MGMT by promoter methylation is a favorable prognostic marker for patients with HGG and confers sensitivity to chemotherapy, although this has been contested. . $^{12,18,29,38,39}$ In the clinical setting, patients with similar $M G M T$ promoter methylation status have variable prognoses and responses to treatment, underscoring the need to refine its use as a predictive marker. In this study, patients with HGG were stratified into 4 subgroups according to $M G M T$ promoter methylation status and HSPB11 expression levels. Patients with a methylated $M G M T$ promoter and low levels of HSPB11 expression had longer OS and PFS than other patient subgroups. Only patients with a methylated $M G M T$ promoter had a prognosis that differed according to level of HSPB11 expression.

It was hypothesized that the different prognoses of patients with $M G M T$ promoter methylation reflected variable responses to specific treatments. Patients with low HSPB11 expression levels had different prognoses depending upon treatment with combined radiochemotherapy or radiotherapy only, with those receiving the former surviving longer than those receiving the latter. However, for patients who expressed high levels of $H S P B 11$, the prognosis was similar for the 2 treatment strategies, suggesting that high HSPB11 expression is an indicator of chemoresistance and can explain the unfavorable prognosis for some patients with a methylated $M G M T$ promoter. Genes related to the cell cycle and the maintenance of genomic integrity were enriched in patients with HGG who had MGMT promoter methylation and high levels of HSPBII expression. The close functional association between HSPB11 level and genomic maintenance indicates that even with promoter methylation, the response to DNA damage from alkylating agents and the relevant genomic integrity may also modulate the beneficial effect. ${ }^{7}$ We propose that HSPB11 can distinguish between responses to treatment in patients with MGMT promoter methylation due to the functional correlation with the maintenance of genomic integrity and cell cycle regulation; however, the detailed mechanism needs confirmation from in vivo and in vitro experiments. The results of this study indicate that HSPB11 expression level in conjunction with $M G M T$ promoter methylation status can be used to identify patients who are suitable for combined radiochemotherapy. In addition, the HSPB11 expression profile can explain why a subset of patients with a methylated $M G M T$ promoter are resistant to alkylating agents.

\section{Conclusions}

The expression of HSPBII is upregulated in patients with HGG and is independently associated with poor prognosis. HSPB11 expression level can be used in conjunction with $M G M T$ promoter methylation status to identify patients with HGG who can benefit from combined radiochemotherapy. Thus, HSPB11 is a novel biomarker and potential drug target with important therapeutic implications. 


\section{Acknowledgments}

This work was supported by grants from the National High Technology Research and Development Program of China (863) (No. 2012AA02A508), the National Natural Science Foundation of China (No. 81172409), and the Science and Technology Department of Liaoning Province (No. 2011225034).

\section{References}

1. Aoyama A, Steiger RH, Fröhli E, Schäfer R, von Deimling A, Wiestler OD, et al: Expression of alpha B-crystallin in human brain tumors. Int J Cancer 55:760-764, 1993

2. Arrigo AP: In search of the molecular mechanism by which small stress proteins counteract apoptosis during cellular differentiation. J Cell Biochem 94:241-246, 2005

3. Balss J, Meyer J, Mueller W, Korshunov A, Hartmann C, von Deimling A: Analysis of the IDH1 codon 132 mutation in brain tumors. Acta Neuropathol 116:597-602, 2008

4. Bellyei S, Szigeti A, Boronkai A, Pozsgai E, Gomori E, Melegh B, et al: Inhibition of cell death by a novel $16.2 \mathrm{kD}$ heat shock protein predominantly via Hsp90 mediated lipid rafts stabilization and Akt activation pathway. Apoptosis 12:97112, 2007

5. Bellyei S, Szigeti A, Pozsgai E, Boronkai A, Gomori E, Hocsak E, et al: Preventing apoptotic cell death by a novel small heat shock protein. Eur J Cell Biol 86:161-171, 2007

6. Calderwood SK, Khaleque MA, Sawyer DB, Ciocca DR: Heat shock proteins in cancer: chaperones of tumorigenesis. Trends Biochem Sci 31:164-172, 2006

7. Cheng W, Li M, Cai J, Wang K, Zhang C, Bao Z, et al: HDAC4, a prognostic and chromosomal instability marker, refines the predictive value of MGMT promoter methylation. J Neurooncol 122:303-312, 2015

8. Clark JI, Muchowski PJ: Small heat-shock proteins and their potential role in human disease. Curr Opin Struct Biol 10:52-59, 2000

9. Colvin M, Hilton J: Pharmacology of cyclophosphamide and metabolites. Cancer Treat Rep 65 (Suppl 3):89-95, 1981

10. Cornford PA, Dodson AR, Parsons KF, Desmond AD, Woolfenden A, Fordham M, et al: Heat shock protein expression independently predicts clinical outcome in prostate cancer. Cancer Res 60:7099-7105, 2000

11. Ehrnsperger M, Gräber S, Gaestel M, Buchner J: Binding of non-native protein to Hsp25 during heat shock creates a reservoir of folding intermediates for reactivation. EMBO J 16:221-229, 1997

12. Esteller M, Garcia-Foncillas J, Andion E, Goodman SN, Hidalgo OF, Vanaclocha V, et al: Inactivation of the DNA-repair gene MGMT and the clinical response of gliomas to alkylating agents. N Engl J Med 343:1350-1354, 2000

13. Garrido C, Brunet M, Didelot C, Zermati Y, Schmitt E, Kroemer G: Heat shock proteins 27 and 70: anti-apoptotic proteins with tumorigenic properties. Cell Cycle 5:2592-2601, 2006

14. Giese A, Kucinski T, Knopp U, Goldbrunner R, Hamel W, Mehdorn HM, et al: Pattern of recurrence following local chemotherapy with biodegradable carmustine (BCNU) implants in patients with glioblastoma. J Neurooncol 66:351360, 2004

15. Glioma Meta-Analysis Trialists (GMT) Group: Chemotherapy for high-grade glioma. Cochrane Database Syst Rev 4:CD003913, 2002

16. Hartl FU, Hayer-Hartl M: Converging concepts of protein folding in vitro and in vivo. Nat Struct Mol Biol 16:574-581, 2009

17. Haslbeck M, Franzmann T, Weinfurtner D, Buchner J: Some like it hot: the structure and function of small heat-shock proteins. Nat Struct Mol Biol 12:842-846, 2005

18. Hegi ME, Diserens AC, Gorlia T, Hamou MF, de Tribolet N,
Weller M, et al: MGMT gene silencing and benefit from temozolomide in glioblastoma. N Engl J Med 352:997-1003, 2005

19. Hermisson M, Strik H, Rieger J, Dichgans J, Meyermann R, Weller M: Expression and functional activity of heat shock proteins in human glioblastoma multiforme. Neurology 54:1357-1365, 2000

20. Hitotsumatsu T, Iwaki T, Fukui M, Tateishi J: Distinctive immunohistochemical profiles of small heat shock proteins (heat shock protein 27 and alpha B-crystallin) in human brain tumors. Cancer 77:352-361, 1996

21. Hsu AL, Murphy CT, Kenyon C: Regulation of aging and age-related disease by DAF-16 and heat-shock factor. Science 300:1142-1145, 2003

22. Huang W, Sherman BT, Lempicki RA: Systematic and integrative analysis of large gene lists using DAVID bioinformatics resources. Nat Protoc 4:44-57, 2009

23. Ischia J, So AI: The role of heat shock proteins in bladder cancer. Nat Rev Urol 10:386-395, 2013

24. Kamradt MC, Lu M, Werner ME, Kwan T, Chen F, Strohecker A, et al: The small heat shock protein alpha B-crystallin is a novel inhibitor of TRAIL-induced apoptosis that suppresses the activation of caspase-3. J Biol Chem 280:11059-11066, 2005

25. Kaufmann SH: Heat shock proteins and the immune response. Immunol Today 11:129-136, 1990

26. Langer T, Lu C, Echols H, Flanagan J, Hayer MK, Hartl FU: Successive action of DnaK, DnaJ and GroEL along the pathway of chaperone-mediated protein folding. Nature 356:683-689, 1992

27. Latchman DS: Protection of neuronal and cardiac cells by HSP27. Prog Mol Subcell Biol 28:253-265, 2002

28. Louis DN, Ohgaki H, Wiestler OD, Cavenee WK, Burger PC, Jouvet A, et al: The 2007 WHO classification of tumours of the central nervous system. Acta Neuropathol 114:97-109, 2007

29. Pegg AE: Repair of O(6)-alkylguanine by alkyltransferases. Mutat Res 462:83-100, 2000

30. Pozsgai E, Gomori E, Szigeti A, Boronkai A, Gallyas F Jr, Sumegi B, et al: Correlation between the progressive cytoplasmic expression of a novel small heat shock protein (Hsp16.2) and malignancy in brain tumors. BMC Cancer 7:233, 2007

31. Sreedhar AS, Csermely P: Heat shock proteins in the regulation of apoptosis: new strategies in tumor therapy: a comprehensive review. Pharmacol Ther 101:227-257, 2004

32. Stege GJ, Renkawek K, Overkamp PS, Verschuure P, van Rijk AF, Reijnen-Aalbers A, et al: The molecular chaperone alphaB-crystallin enhances amyloid beta neurotoxicity. Biochem Biophys Res Commun 262:152-156, 1999

33. Strik HM, Weller M, Frank B, Hermisson M, Deininger MH, Dichgans J, et al: Heat shock protein expression in human gliomas. Anticancer Res 20:4457-4462, 2000

34. Stupp R, Brada M, van den Bent MJ, Tonn JC, Pentheroudakis G: High-grade glioma: ESMO Clinical Practice Guidelines for diagnosis, treatment and follow-up. Ann Oncol 25 (Suppl 3):iii93-iii101, 2014

35. Stupp R, Mason WP, van den Bent MJ, Weller M, Fisher $\mathrm{B}$, Taphoorn MJ, et al: Radiotherapy plus concomitant and adjuvant temozolomide for glioblastoma. $\mathbf{N}$ Engl J Med 352:987-996, 2005

36. Subramanian A, Tamayo P, Mootha VK, Mukherjee S, Ebert BL, Gillette MA, et al: Gene set enrichment analysis: a knowledge-based approach for interpreting genome-wide expression profiles. Proc Natl Acad Sci U S A 102:1554515550,2005

37. Turcan S, Rohle D, Goenka A, Walsh LA, Fang F, Yilmaz E, et al: IDH1 mutation is sufficient to establish the glioma hypermethylator phenotype. Nature 483:479-483, 2012 
38. van den Bent MJ, Dubbink HJ, Sanson M, van der Lee-Haarloo CR, Hegi M, Jeuken JW, et al: MGMT promoter methylation is prognostic but not predictive for outcome to adjuvant PCV chemotherapy in anaplastic oligodendroglial tumors: a report from EORTC Brain Tumor Group Study 26951. J Clin Oncol 27:5881-5886, 2009

39. Weller M, Stupp R, Reifenberger G, Brandes AA, van den Bent MJ, Wick W, et al: MGMT promoter methylation in malignant gliomas: ready for personalized medicine? Nat Rev Neurol 6:39-51, 2010

40. Wick W, Hartmann C, Engel C, Stoffels M, Felsberg J, Stockhammer F, et al: NOA-04 randomized phase III trial of sequential radiochemotherapy of anaplastic glioma with procarbazine, lomustine, and vincristine or temozolomide. J Clin Oncol 27:5874-5880, 2009

41. Wick W, Platten M, Meisner C, Felsberg J, Tabatabai G, Simon M, et al: Temozolomide chemotherapy alone versus radiotherapy alone for malignant astrocytoma in the elderly: the NOA-08 randomised, phase 3 trial. Lancet Oncol 13:707-715, 2012

42. Yan H, Parsons DW, Jin G, McLendon R, Rasheed BA, Yuan W, et al: IDH1 and IDH2 mutations in gliomas. N Engl J Med 360:765-773, 2009

43. Yan W, Zhang W, You G, Bao Z, Wang Y, Liu Y, et al: Correlation of IDH1 mutation with clinicopathologic factors and prognosis in primary glioblastoma: a report of 118 patients from China. PLoS One 7:e30339, 2012

44. Zhang C, Bao Z, Zhang W, Jiang T: Progress on molecular biomarkers and classification of malignant gliomas. Front Med 7:150-156, 2013

45. Zhang CB, Bao ZS, Wang HJ, Yan W, Liu YW, Li MY, et al: Correlation of IDH1/2 mutation with clinicopathologic factors and prognosis in anaplastic gliomas: a report of 203 patients from China. J Cancer Res Clin Oncol 140:45-51, 2014
46. Zhao D, Jiang X, Yao C, Zhang L, Liu H, Xia H, et al: Heat shock protein 47 regulated by miR-29a to enhance glioma tumor growth and invasion. J Neurooncol 118:39-47, 2014

47. Zoubeidi A, Gleave M: Small heat shock proteins in cancer therapy and prognosis. Int J Biochem Cell Biol 44:16461656,2012

\section{Disclosure}

The authors report no conflict of interest concerning the materials or methods used in this study or the findings specified in this paper.

\section{Author Contributions}

Conception and design: $\mathrm{Wu}$, Cheng. Analysis and interpretation of data: Cheng. Drafting the article: Cheng. Statistical analysis: Cheng, Li, Zhang, Cai, Wang. Administrative/technical/material support: Jiang. Study supervision: Li, Zhang, Cai.

\section{Supplemental Information}

Online-Only Content

Supplemental material is available with the online version of the article.

Supplemental Tables S1-S5 and Supplemental Figures S1-S6. http://thejns.org/doi/suppl/10.3171/2015.5.JNS142437.

\section{Correspondence}

Anhua Wu, Department of Neurosurgery, The First Hospital of China Medical University, Nanjing Street 155, Heping District, Shenyang 110001, China. email: cmuwuanhua@aliyun.com. 